\title{
Fluorine nucleosynthesis and s-processing in AGB stars driven by magnetic-buoyancy mixing
}

\author{
Diego Vescovi ${ }^{1, *}$, Sergio Cristallo ${ }^{2,3}$, Sara Palmerini ${ }^{3,4}$, Carlos Abia ${ }^{5}$, and Maurizio Busso ${ }^{3,4}$ \\ ${ }^{1}$ Goethe University Frankfurt, Max-von-Laue-Strasse 1, Frankfurt am Main 60438, Germany \\ ${ }^{2}$ INAF, Observatory of Abruzzo, Via Mentore Maggini snc, 64100 Teramo, Italy \\ ${ }^{3}$ INFN, Section of Perugia, Via A. Pascoli snc, 06123 Perugia, Italy \\ ${ }^{4}$ Department of Physics and Geology, University of Perugia, via A. Pascoli snc, 06123 Perugia, Italy \\ ${ }^{5}$ University of Granada, Departamento de Fisica Teorica y del Cosmos, 18071 Granada, Spain
}

\begin{abstract}
Asymptotic giant branch (AGB) stars are thought to be among the most important sources of fluorine in our Galaxy. While observations and theory agree at close-to-solar metallicity, stellar models overestimate fluorine production in comparison to heavy elements at lower metallicities. We present predictions for ${ }^{19} \mathrm{~F}$ abundance for a set of AGB models with various masses and metallicities, in which magnetic buoyancy induces the formation of the ${ }^{13} \mathrm{C}$ neutron source (the so-called ${ }^{13} \mathrm{C}$ pocket). In our new models, fluorine is mostly created as a consequence of secondary ${ }^{14} \mathrm{~N}$ nucleosynthesis during convective thermal pulses, with a minor contribution from the ${ }^{14} \mathrm{~N}$ existing in the ${ }^{13} \mathrm{C}$ pocket zone. As a result, AGB stellar models with magnetic-buoyancyinduced mixing show low ${ }^{19} \mathrm{~F}$ surface abundances which agree with fluorine spectroscopic observations at both low and near-solar metallicity.
\end{abstract}

\section{Introduction}

The cosmic genesis of fluorine is one of the most intriguing problems in nuclear astrophysics. So far, spectroscopic observations of photospheric F enhancements in intrinsic AGB carbon stars [1,2] and metal-poor extrinsic stars [3, 4] offer the sole direct observation of fluorine production. Its envelope abundance indicates a correlation with those of carbon and $s$-process elements and therefore with the ${ }^{13} \mathrm{C}$ content produced in the interiors of AGB stars [5]. Recently, it was proposed that a process resulting in the creation of an extended ${ }^{13} \mathrm{C}$ pocket and, simultaneously, a tiny quantity of ${ }^{14} \mathrm{~N}$, may solve the issue of ${ }^{19} \mathrm{~F}$ overproduction with respect to s-elements in low-mass metal-poor objects [4].

The formation of a ${ }^{13} \mathrm{C}$ pocket in AGB stars requires that some partial mixing of protons from the envelope occurs during a third dredge-up (TDU) phenomenon. In latest FRUITY models [6], the buoyant rise of magnetic flux tubes in the region below the envelope is considered to cause a stable mass upflow which induces a downflow of protons for maintaining mass conservation. As a result of such magnetic mixing, deep profiles of low proton abundances are predicted to be formed. Because protons are nearly completely used for the synthesis of ${ }^{13} \mathrm{C}$, the low proton concentration significantly reduces the local ${ }^{14} \mathrm{~N}$ formation, thereby limiting ${ }^{19} \mathrm{~F}$ production as well. Here we examine fluorine nucleosynthesis in low-mass AGB

\footnotetext{
*e-mail: vescovi@iap.uni-frankfurt.de
} 

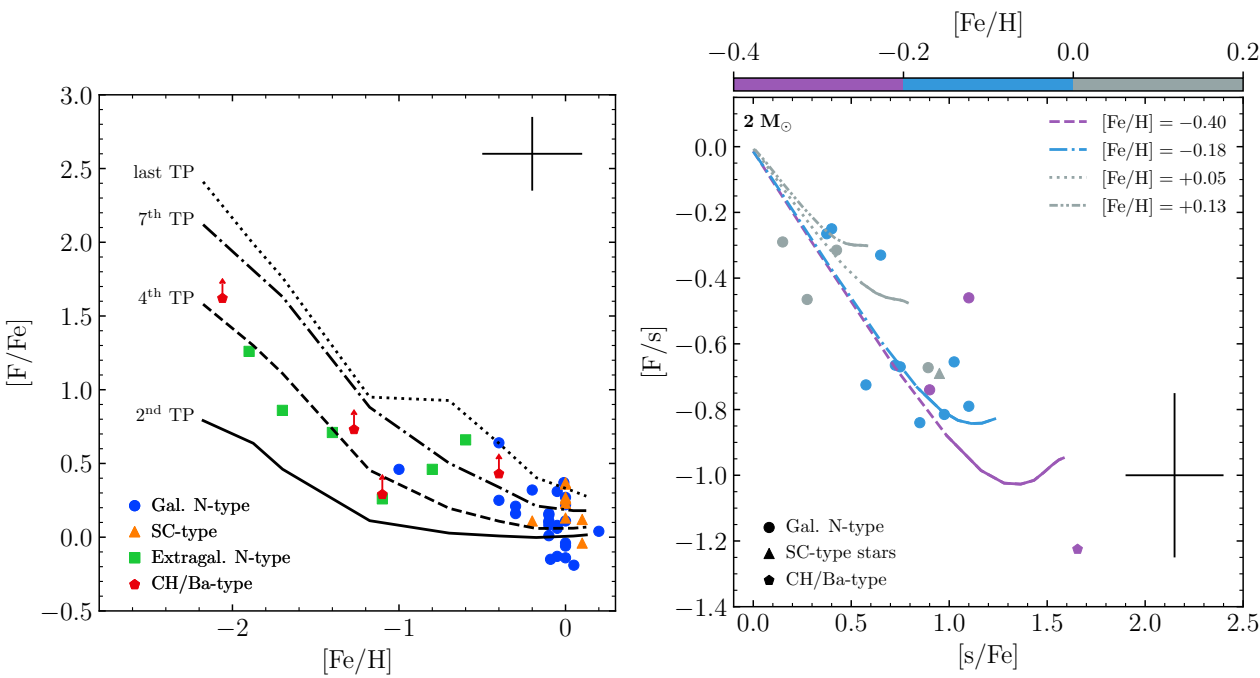

Figure 1. (Left panel) Comparison between observed $[\mathrm{F} / \mathrm{Fe}]$ ratios as a function of the metallicity and FRUITY magnetic models. Symbols refer to four data groups: circles, galactic (N-type) carbon stars; triangles, SC-type stars; squares, extragalactic carbon stars; pentagons, extrinsic $\mathrm{CH} / \mathrm{Ba}$ stars. Lines represent theoretical predictions for 2 (for $[\mathrm{Fe} / \mathrm{H}] \geq-0.7)$ and $1.5 M_{\odot}($ for $[\mathrm{Fe} / \mathrm{H}]<-0.7)$ AGB stars at different thermal pulses (TPs). (Right panel) Observed $[\mathrm{F} / \mathrm{s}]$ vs. average s-element enhancements compared with theoretical predictions for $2 M_{\odot}$ and close-to-solar metallicity models. Data points and theoretical lines are color-coded by $[\mathrm{Fe} / \mathrm{H}]$. The solid portion of the lines represents the theoretical $\mathrm{C}$-rich phase, while the dashed portion represents the O-rich phase. Typical error bars are indicated.

stars by computing a new series of stellar models accounting for the formation of a magneticbuoyancy-induced ${ }^{13} \mathrm{C}$ pocket.

\section{Comparison with observations}

In the following, we compare data for intrinsic AGB carbon stars $[2,4]$ and extrinsic $\mathrm{CH} / \mathrm{Ba}$ stars $[4,5]$ with new FRUITY magnetic models of $1.5 M_{\odot}$ and $2 M_{\odot}$.

In left panel of Fig. 1 we report the $[\mathrm{F} / \mathrm{Fe}]$ ratios of the selected sample as a function of $[\mathrm{Fe} / \mathrm{H}]$. Within the observational errors, there is a good agreement, confirming the expected F-enhancement trend with the metallicity. The right panel of Fig. 1 shows the comparison between theoretical predictions of FRUITY magnetic models and spectroscopic observations for $[\mathrm{F} / \mathrm{s}]$ ratios vs. the average s-element enhancement. In this way, the $\mathrm{F}$ enhancement for the extrinsic stars is not affected by uncertainties related to the dilution factor and provides a robust tool for comparison. Theoretical expectations can replicate the quasi-linear decreasing trend of $[\mathrm{F} / \mathrm{s}]$ with the surface s-process enrichment.

In Fig. 2 we perform a similar comparison at low metallicities. We compared model predictions individually since there is no homogeneous sample of stars with both $\mathrm{Ba}$ and $\mathrm{La}$. Magnetic models well reproduce the spread observed at different metallicities for both $[\mathrm{F} / \mathrm{Ba}]$ and $[\mathrm{F} / \mathrm{La}]$ ratios as a function of total s-process enhancement. Overall, FRUITY magnetic models show a reduction in fluorine production that is consistent with spectroscopic observations of low-metallicity stars. The extended profile and low proton abundance that characterize FRUITY magnetic models [6] have the dual impact of generating large ${ }^{13} \mathrm{C}$ pockets and a small amount of primary ${ }^{14} \mathrm{~N}$. In this scenario, the few available protons make 

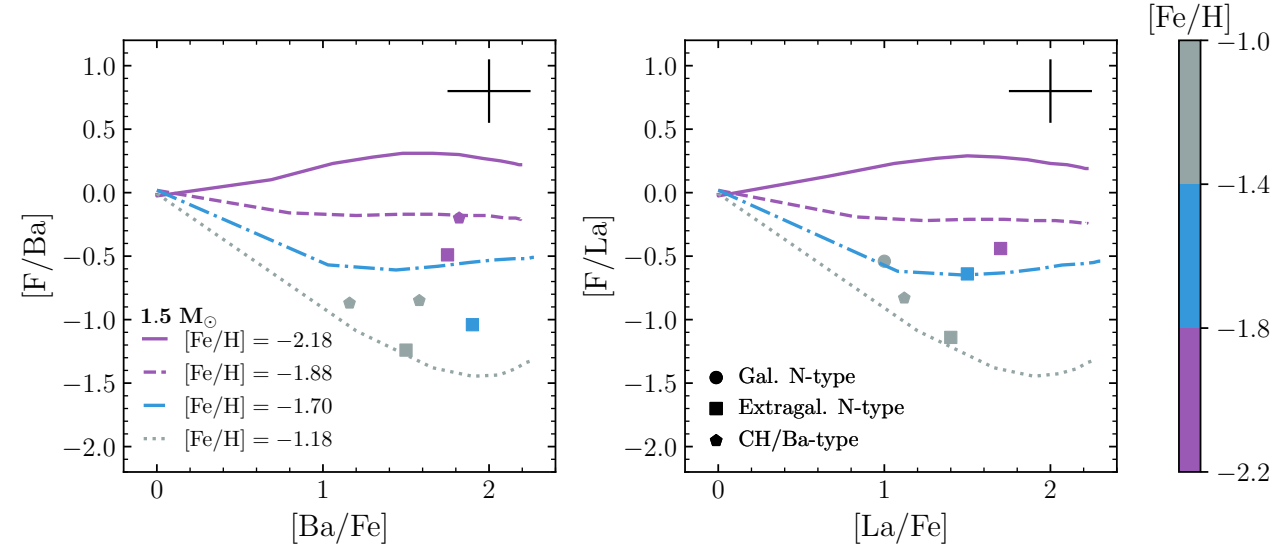

Figure 2. $[\mathrm{F} / \mathrm{Ba}]$ vs. $[\mathrm{Ba} / \mathrm{Fe}]$ (left panel) and $[\mathrm{F} / \mathrm{La}]$ vs. $[\mathrm{La} / \mathrm{Fe}]$ (right panel) in the sample stars with $[\mathrm{Fe} / \mathrm{H}] \leq-1.0$. Symbols as in Fig. 1. Note that at these low metallicities, theoretical AGB models predict that the star becomes C-rich from first TDU episodes. Data points and theoretical lines are color-coded by $[\mathrm{Fe} / \mathrm{H}]$. Typical error bars are indicated.

first ${ }^{13} \mathrm{C}$ through the ${ }^{12} \mathrm{C}(\mathrm{p}, \gamma){ }^{13} \mathrm{C}$ reaction, preventing further proton captures to form ${ }^{14} \mathrm{~N}$, thus inhibiting the nuclear chain ${ }^{14} \mathrm{~N}(\mathrm{n}, \mathrm{p}){ }^{14} \mathrm{C}(\alpha, \gamma){ }^{18} \mathrm{O}(\mathrm{p}, \alpha){ }^{15} \mathrm{~N}(\alpha, \gamma){ }^{19} \mathrm{~F}$. As a consequence, any fluorine appearing in AGB envelopes in these models is of secondary origin, caused by ${ }^{14} \mathrm{~N}$ concentrations left over after $\mathrm{H}$-shell burning. As an outcome, FRUITY magnetic models have low fluorine enhancements and large s-enhancements, which are in close agreement with observations in very metal-poor AGB stars [7].

\section{Conclusions}

The production of fluorine in low-mass AGB stars has been examined in light of new FRUITY stellar models, in which the ${ }^{13} \mathrm{C}$ neutron source is attributed to magnetic-buoyancy-induced phenomena. On the one hand, the new FRUITY magnetic models exhibit a low net ${ }^{19} \mathrm{~F}$ production. This is due to the low abundance of ${ }^{14} \mathrm{~N}$ in the ${ }^{13} \mathrm{C}$ pocket, which results in negligible fluorine production during ${ }^{13} \mathrm{C}$ radiative burning. The ${ }^{19} \mathrm{~F}$ envelope abundance is therefore ascribed only to the amount of the secondary ${ }^{13} \mathrm{C}$ in the $\mathrm{H}$-shell ashes, which depends on the $\mathrm{CNO}$ abundances in the star. On the other hand, mixing induced by magnetic buoyancy leads to extended ${ }^{13} \mathrm{C}$ pockets so resulting in large surface s-process enrichments. As a whole, new FRUITY magnetic models simultaneously account for both the observed fluorine and the average s-element enhancements in intrinsic AGB carbon stars and extrinsic $\mathrm{CH} / \mathrm{Ba}$ stars.

\section{References}

[1] A. Jorissen, V.V. Smith, D.L. Lambert, A\&A 261, 164 (1992)

[2] C. Abia, K. Cunha, S. Cristallo, P. de Laverny, A\&A 581, A88 (2015)

[3] S. Lucatello, T. Masseron, J.A. Johnson, M. Pignatari, F. Herwig, ApJ 729, 40 (2011)

[4] C. Abia, S. Cristallo, K. Cunha, P. de Laverny, V.V. Smith, A\&A 625, A40 (2019)

[5] M. Forestini, S. Goriely, A. Jorissen, M. Arnould, A\&A 261, 157 (1992)

[6] D. Vescovi, S. Cristallo, M. Busso, N. Liu, ApJL 897, L25 (2020)

[7] D. Vescovi, S. Cristallo, S. Palmerini, C. Abia, M. Busso, A\&A 652, A100 (2021) 